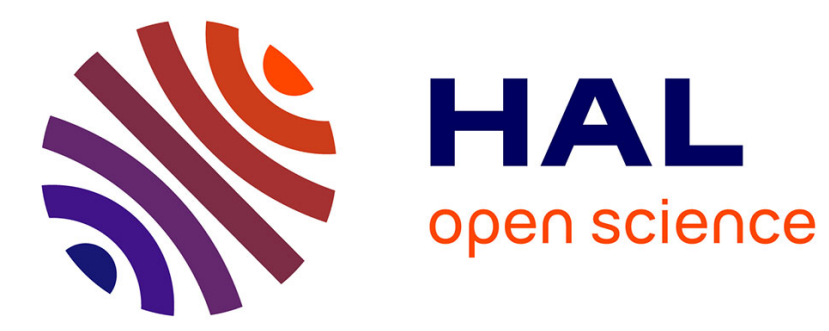

\title{
Les groupes préhispaniques du littoral Pacifique nord-équatorial : un cas de peuplement tardif en Amérique du Sud
}

Jean-François Bouchard

\section{- To cite this version:}

Jean-François Bouchard. Les groupes préhispaniques du littoral Pacifique nord-équatorial: un cas de peuplement tardif en Amérique du Sud. Bulletin de la S. P. F. T., 1994, 4-5, pp.316-323. 10.3406/bspf.1994.9740 . hal-02891864

\section{HAL Id: hal-02891864 https://hal.science/hal-02891864}

Submitted on 8 Jul 2020

HAL is a multi-disciplinary open access archive for the deposit and dissemination of scientific research documents, whether they are published or not. The documents may come from teaching and research institutions in France or abroad, or from public or private research centers.
L'archive ouverte pluridisciplinaire HAL, est destinée au dépôt et à la diffusion de documents scientifiques de niveau recherche, publiés ou non, émanant des établissements d'enseignement et de recherche français ou étrangers, des laboratoires publics ou privés. 
Les groupes préhispaniques du littoral pacifique nord-équatorial : un cas de peuplement tardif en Amérique du Sud Jean-François Bouchard

\section{Citer ce document / Cite this document :}

Bouchard Jean-François. Les groupes préhispaniques du littoral pacifique nord-équatorial : un cas de peuplement tardif en Amérique du Sud. In: Bulletin de la Société préhistorique française, tome 91, n4-5, 1994. Le peuplement préhistorique de l'Amérique. pp. 316-323;

doi : https://doi.org/10.3406/bspf.1994.9740

https://www.persee.fr/doc/bspf_0249-7638_1994_num_91_4_9740

Fichier pdf généré le 10/01/2019 


\section{Resumen}

RESUMEN El litoral Pacifico nor ecuatorial constituye un ejemplo de pobla- miento tardio de regiones que habian permanecido inhabitadas por largo tiempo antes que llegaran a asen- tarse alii migraciones proveniendo desde costas cercanas. Para poder asentarse de forma durable, estos grupos tuvieron que adaptarse a un medio ambiente muy peculiar, en el cual el papel del agua es prédominante. El oceano y los ríos del litoral les permitían desplazarse sobre el agua con mayor facilidad que sobre la tierra, densamente cubierta de selvas pantanosas, a la vez que les proporcionaban varios e importantes recursos alimenticios para comple- mentar su producción agrícola y la recolección de plantas y frutos sil- vestres. Por lo tanto, dichos grupos tenian que dominar las técnicas de navega- ción indispensables tanto para sus contactos culturales como para su economia. Este durable desarrollo cultural représenta un caso muy interesante para un modelo que se acerca más a los modelos conocidos para las altas culturas andinas que a los modelos que suelen caracterizar los grupos de selva tropical humeda en America de Sur, aunque el medio natural corresponde más bien a los que se en- cuentran en las tierras bajas y selváticas.

\section{Résumé}

RÉSUMÉ Le littoral Pacifique nord-équato- rial constitue un exemple de peuplement tardif de contrées qui demeurèrent longtemps inhabitées et qui furent atteintes par des migrations venues de régions voisines. Pour s'établir durablement dans cette région, ces groupes durent s'adapter à un environnement particulier, dans lequel le milieu aquatique joue un rôle majeur. L'océan et les cours d'eau du littoral permirent à la fois à ces populations de se déplacer beaucoup plus aisément que par voie terrestre, au milieu d'une forêt dense et de zones marécageuses, et de se procurer par la pêche d'importantes ressources alimentaires complétant la production agricole et la cueillette d'aliments végétaux sauvages. Ces groupes durent ainsi maîtriser les techniques de navigation qui leur permettaient d'établir les contacts indispensables à leur économie et à leur culture. Le développement durable de cette civilisation constitue un exemple intéressant d'un modèle plus proche des "hautes cultures an- dines" que des cultures des forêts tropicales des basses terres de l'Amérique du Sud, bien que l'environnement ait été assez comparable à celui des selvas.

\footnotetext{
Abstract

ABSTRACT The northern equatorial Pacific coast of America remained uninhabited for a long period of time until human groups, migrating from nearby coasts, settled there only quite recently. In order to survive, these first groups had to adapt themselves to semi-aquatic environmental conditions. Although they planted and gathered vegetal food, they could also have acquired large amounts of food through fishing at sea or in the rivers, estuaries or tidal channels. Therefore, they had to develop efficient maritime and fluvial navigation tech- niques which were essential to their mid and long range economic and cultural trading. Such a long lasting cultural development appears to be a special and interesting model which is more comparable to the models known for the prehispanic Andean cultures than to the models known for tropical rain forest cultures, in spite of the similar environmental conditions shared by these tropical forest groups.
} 


\title{
LES GROUPES PRÉHISPANIQUES DU LITTORAL PACIFIQUE NORD-ÉQUATORIAL : UN CAS DE PEUPLEMENT TARDIF EN AMÉRIQUE DU SUD
}

\author{
Jean-François BOUCHARD
}

\section{RÉSUMÉ}

Le littoral Pacifique nord-équatorial constitue un exemple de peuplement tardif de contrées qui demeurèrent longtemps inhabitées et qui furent atteintes par des migrations venues de régions voisines.

Pour s'établir durablement dans cette région, ces groupes durent s'adapter à un environnement particulier, dans lequel le milieu aquatique joue un rôle majeur. L'océan et les cours d'eau du littoral permirent à la fois à ces populations de se déplacer beaucoup plus aisément que par voie terrestre, au milieu d'une forêt dense et de zones marécageuses, et de se procurer par la pêche d'importantes ressources alimentaires complétant la production agricole et la cueillette d'aliments végétaux sauvages.

Ces groupes durent ainsi maîtriser les techniques de navigation qui leur permettaient d'établir les contacts indispensables à leur économie et à leur culture.

Le développement durable de cette civilisation constitue un exemple intéressant d'un modèle plus proche des "hautes cultures andines" que des cultures des forêts tropicales des basses terres de l'Amérique du Sud, bien que l'environnement ait été assez comparable à celui des selvas.

\section{ABSTRACT}

The northern equatorial Pacific coast of America remained uninhabited for a long period of time until human groups, migrating from nearby coasts, settled there only quite recently. In order to survive, these first groups had to adapt themselves to semi-aquatic environmental conditions.

Although they planted and gathered vegetal food, they could also have acquired large amounts of food through fishing at sea or in the rivers, estuaries or tidal channels. Therefore, they had to develop efficient maritime and fluvial navigation tech- niques which were essential to their mid and long range economic and cultural trading.

Such a long lasting cultural development appears to be a special and interesting model which is more comparable to the models known for the prehispanic Andean cultures than to the models known for tropical rain forest cultures, in spite of the similar environmental conditions shared by these tropical forest groups.

\section{RESUMEN}

El litoral Pacífico nor ecuatorial constituye un ejemplo de poblamiento tardío de regiones que habian permanecido inhabitadas por largo tiempo antes que llegaran a asentarse allí migraciones proveniendo desde costas cercanas.

Para poder asentarse de forma durable, estos grupos tuvieron que adaptarse a un medio ambiente muy peculiar, en el cual el papel del agua es predominante.

El oceano y los ríos del litoral les permitían desplazarse sobre el agua con mayor facilidad que sobre la tierra, densamente cubierta de selvas pantanosas, a la vez que les proporcionaban varios e importantes recursos alimenticios para complementar su producción agrícola y la recolección de plantas y frutos silvestres.

Por lo tanto, dichos grupos tenían que dominar las técnicas de navegación indispensables tanto para sus contactos culturales como para su economía.

Este durable desarrollo cultural representa un caso muy interesante para un modelo que se acerca más a los modelos conocidos para las altas culturas andinas que a los modelos que suelen caracterizar los grupos de selva tropical húmeda en America de Sur, aunque el medio natural corresponde más bien a los que se encuentran en las tierras bajas y selváticas.

\section{LE MILIEU GÉOGRAPHIQUE : MANGROVE ET FORÊT ÉQUATORIALE}

Entre le $1^{\circ}$ et le $3^{\circ}$ de latitude Nord, la région côtière nord-équatoriale est constituée par une vaste plaine, large d'une cinquantaine de $\mathrm{km}$, située entre l'Océan Pacifique et la cordillère occidentale. Une coupe transversale ouest/est révèle, dans la quasi-totalité des cas, une première frange côtière, composée d'une forêt de palétuviers, de marécages ou de lagunes. Elle peut s'étendre sur plusieurs kilomètres de large et borde le cours inférieur des fleuves qui descendent de la cordillère. A mer haute, les chenaux de marée, ou esteros, forment un dédale d'affluents temporaires des cours d'eau réguliers. Tout autour, s'étend la mangrove dont les sols sont submergés par les hautes eaux. Seules quelques berges plus élevées, les firmes, permettent d'y établir des habitations. Les études palynologiques ont démontré la stabilité globale de ces paysages au cours des trois derniers millénaires. Au-delà, commence la "terre ferme", formée d'une basse plaine alluviale recouverte de forêt tropicale humide, jusqu'au piémont de la cordillère orientale, où la végétation de forêt tropicale se modifie progressivement avec l'altitude.

La région des basses terres du $\mathrm{Pa}$ cifique nord-équatorial peut aussi se décomposer en trois secteurs distincts qui diffèrent de la traditionnelle classification qui oppose la frange côtière, la plaine alluviale et le piémont.

Il est ainsi possible de reconnaître un secteur aquatique et un secteur terrestre, entre lesquels s'interpose un secteur mixte qui participe des deux éléments liquide et solide. Ces trois secteurs furent diversement utilisés et occupés par les groupes humains à l'époque préhispanique comme de nos jours.

Le climat de tout ce littoral est profondément marqué par la proximité de l'océan et par celle de la cor- 


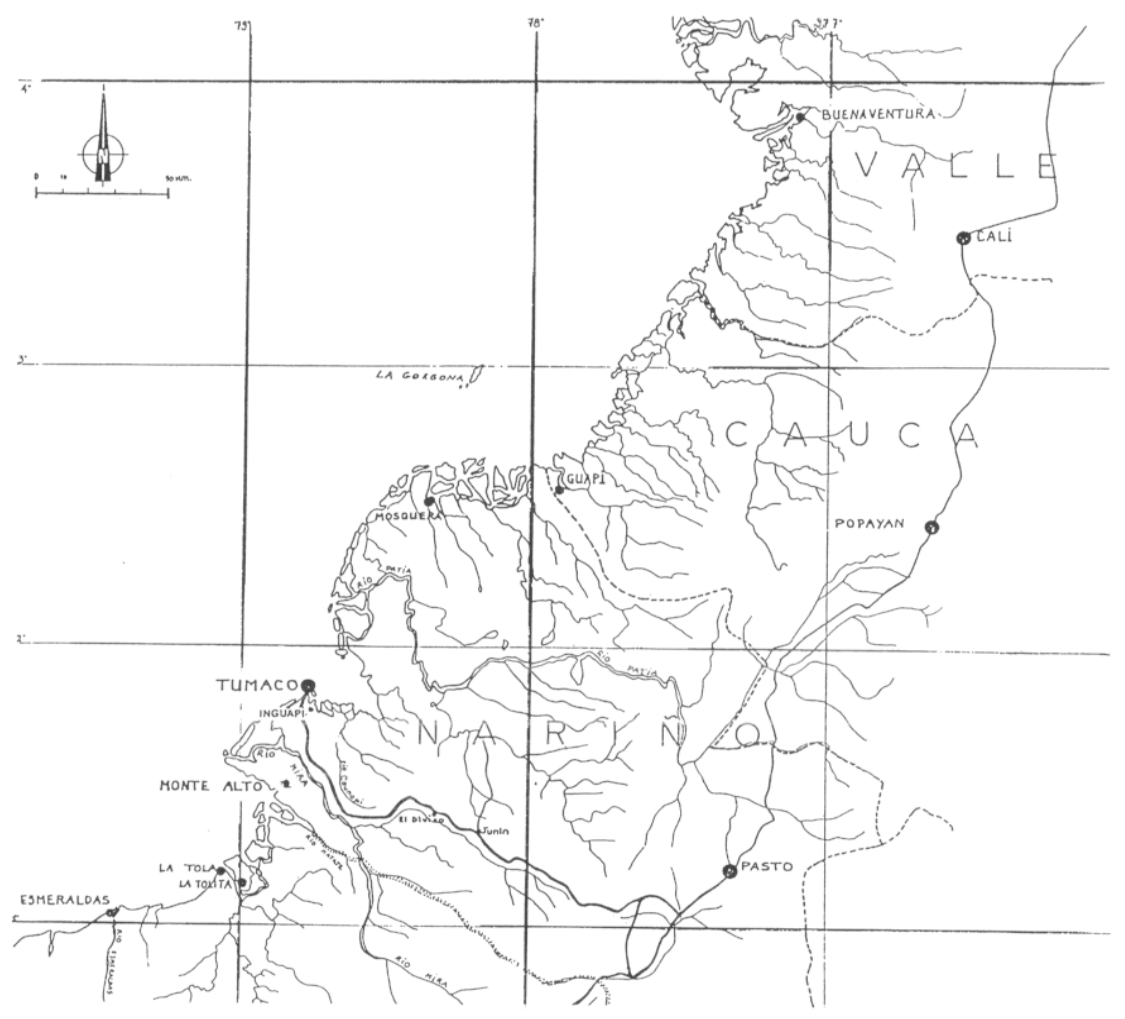

Carte du littoral pacifique nord-équatorial

dillère. II se caractérise par d'abondantes précipitations qui s'abattent toute l'année y compris pendant une saison plus sèche où les pluies diminuent un peu en fréquence et en abondance. Ces importantes précipitations $(2000 \mathrm{~mm} / \mathrm{an}$ sur la côte mais plus de $5000 \mathrm{~mm} / \mathrm{an}$ sur le piémont de la cordillère occidentale) sont dues à la masse de nuages formés par l'évaporation sur l'océan et poussés par les vents dominants sur le littoral où ils sont bloqués par les premières hauteurs des Andes. De telles pluies sont la cause d'une forte érosion et d'un important lessivage des sols qui rendent particulièrement difficiles les conditions de vie et de subsistance en dehors de la frange côtière, un peu moins humide que le reste de la plaine alluviale.

\section{L'ÉVOLUTION CHRONOCULTURELLE RÉGIONALE}

\section{- La Période Formative}

Les recherches archéologiques réalisées dans cette région situent les plus anciens vestiges d'occupa- tion humaine vers 600 av. J.-C. sur la base de plusieurs datations ${ }^{14} \mathrm{C}$. Cela signifie, dans l'hypothèse vraisemblable où aucun vestige plus ancien n'apparaîtrait au cours de futures recherches, que la région demeura inhabitée pendant les millénaires précédents. Dans des régions côtières proches, à partir de 4000-3500 av J.-C. environ, les groupes de la tradition Valdivia produisaient déjà l'une des plus anciennes céramiques du continent (mais peut être pas "initiale"), pratiquaient l'agriculture et formaient des villages complexes. C'est donc au cours de cette période qu'apparut une production qui aurait permis, entre autre, l'émergence de classes spécialisées d'artisans et de dirigeants religieux et politiques (Marcos, 1992).

Une fois assises les bases d'une économie de production excédentaire il se produisit une forte augmentation démographique des noyaux de départ, entraînant un essaimage vers des terres voisines encore non habitées.

Si la sphère d'occupation Valdivia semble assez restreinte et ne touche pas le littoral nord-équatorial, les po- pulations suivantes, possédant une tradition de navigation propre à ces groupes culturels côtiers, se déplacèrent le long des côtes par voie maritime plutôt que d'entreprendre une migration terrestre. Dans la région du littoral nord-équatorial, ce n'est que vers 600 av. J.-C. qu'arrivèrent ces migrations, issues de traditions dont les débuts remontent donc à près de trois millénaires

Les recherches ont permis la découverte de sites anciens littoraux, un peu en retrait des côtes et presque toujours près des cours d'eau, sur les berges que la marée ne recouvre pas. Seuls quelques rares sites ont été découverts plus à l'intérieur, et seulement dans certaines portions de la région littorale (de Boer, Tolstoy, 1989). Pour s'établir, ces groupes préhispaniques choisissaient surtout une position de seuil, près de la mangrove, leur donnant ainsi accès au milieu aquatique et au milieu terrestre.

\section{- La Période des Développements Régionaux}

Cette utilisation et cette occupation de la région littorale nord équatoriale se poursuivit sous une forme analogue après la fin de la période Formative, lors de la Période des Développements Régionaux, qui s'ouvre à partir de 300 av. J.-C. environ et dont la fin peut être située vers 300 ap. J.-C. pour la région de La Tolita-Tumaco. En Équateur, elle se caractérise par la floraison de styles régionaux bien différenciés les uns des autres.

L'interprétation anciennement donnée à ce changement n'est plus vraiment satisfaisante car elle suggérait la disparition des populations antérieures et leur remplacement par des migrations, souvent d'origine lointaine. Au contraire, dans la région nord-équatoriale, on a pu mettre récemment en évidence les liens entre les cultures de la fin de la Période Formative et celles de la Période des Développements Régionaux. La céramique de ces différents niveaux permet de suivre une filiation clairement perceptible dans les récipients aussi bien pour la morphologie que pour les techniques de fabrication ou de décoration.

Toutefois, les groupes culturels paraissent avoir connu une évolution assez importante. Si l'on peut sup- 
poser pour la période antérieure des sociétés dirigées par un fort pouvoir politique et religieux mais dont la population demeurait majoritairement rattachable au secteur primaire, au cours de cette nouvelle période se développent alors des sociétés organisées en chefferies et sans doute plus complexes. L'augmentation démographique permit alors, à côté des catégories du secteur primaire, le développement de catégories sociales liées à l'obtention de diverses matières premières et à leur transformation en produits finis. II y eut aussi un fort développement du secteur tertiaire, lié aux pouvoirs politiques et religieux, et sans doute aussi celui de catégories sociales particulières dédiées aux trafics locaux et aux échanges inter-régionaux.

C'est vraisemblablement pendant cette Période des Développements Régionaux que se constituèrent en tant qu'entités propres les différentes ethnies préhispaniques des Andes équatoriales, faisant suite aux premières individualisations de la Période Formative finale. Cette différenciation se reflète principalement dans la céramique où on observe l'abandon des caractères qui constituaient le tronc commun permettant de relier entre eux, malgré tout, les divers styles régionaux du Formatif final.

Dans le littoral nord-équatorial, la région de La Tolita dût accueillir, à l'époque des "Développements Régionaux", une population particulièrement nombreuse et jouer en même temps un rôle de métropole régionale. On retrouve en effet dans l'ensemble de la région une céramique stylistiquement homogène, malgré quelques variantes spatiales. De même, les figurines en céramique reproduisent, parfois à des centaines d'exemplaires, des personnages humains et des animaux typiques de la faune locale.

Les données obtenues à La Tolita et dans d'autres sites de toute la région côtière, en Équateur comme en Colombie, permettent de proposer une interprétation qui repose aussi sur des observations effectuées à partir de la vie moderne. II semble que se développèrent des agglomérations reprenant le modèle des établissements de la Période antérieure du "Formatif" final. Toujours sur des cours d'eau, ces agglomérations durent jouer alors un rôle de centres principaux reliés à des établissements ruraux de production agricole.
II est vraisemblable que pendant la Période des Développements Régionaux, l'île de La Tolita constitua, une métropole puissante et de type hégémonique, diffusant son influence dans l'ensemble du littoral nord-équatorial (Bouchard, 1894,1986,1989 ; Valdez, 1989).

Cette influence ne paraît pas s'être maintenue aussi fortement jusqu'à la fin de la période des "Développements Régionaux". En effet, vers la fin du $1^{\text {er }}$ siècle de notre ère, dans la céramique apparaissent au nord et au sud des variantes locales ou des innovations (Bouchard, 1984, 1987 ; Patiño Castaño, 1987). Cellesci pourraient indiquer la récession de l'influence de La Tolita, peut-être réduite au bassin du Santiago et n'atteignant plus aussi fortement les autres bassins fluviaux. Ce déclin peut s'expliquer, éventuellement, par le développement de l'indépendance de territoires lointains où la métropole ancienne finit par perdre sa suprématie.

\section{- La Période d'Intégration}

Cette Période des Développements régionaux s'achève dans les diverses régions de l'Équateur entre 300 et 500 après J.-C. En effet, vers cette époque la plupart des cultures régionales disparaissent ou se transforment à nouveau. Dans la région nord-équatoriale, il existe actuellement un profond manque de connaissances pour les siècles qui suivent et qui portent génériquement le nom de Période d'Intégration. En outre, alors que la fin de l'époque préhispanique est, pour la majeure partie de l'Équateur, marquée par l'invasion Inca, le littoral nord-équatorial ne connut pas cette dernière période de l'hégémonie inca.

Les données dont nous disposons sont fragmentaires et parfois contradictoires ou, pour le moins, discordantes. C'est donc un peu plus de dix siècles dont l'histoire ne peut être reconstituée que par bribes et pour lesquels l'archéologie doit faire appel à la logique et à la comparaison plus qu'aux données de fouille pour proposer une suite aux périodes mieux connues bien que plus anciennes.

Parmi les vestiges archéologiques les plus remarquables, on date de cette période la plupart des monticules artificiels, appelés tolas. Beau- coup de ces monticules ont dû servir de bases surélevées pour des constructions domestiques ou cérémonielles. D'autres furent construits pour servir de tertres funéraires. Certains aussi semblent avoir rempli les deux fonctions : d'abord servant de base à des constructions, ils furent réutilisés pour y creuser des sépultures postérieures à la construction des tolas.

Dans la région du littoral pacifique nord-équatorial, les fouilles qui ont porté sur ces monticules, dans la région d'Esmeraldas (en Équateur) comme dans la région de Tumaco (en Colombie), ont donné des datations de ${ }^{14} \mathrm{C}$ correspondant à la Période d'Intégration pour les vestiges contemporains de ces tolas. Dans divers bassins fluviaux, à quelques dizaines de kilomètres, les fouilles ont mis à jour des niveaux préhispaniques datés de cette époque (aux alentours des $10^{\mathrm{e}}, 11^{\mathrm{e}}$ et $12^{\mathrm{e}}$ siècles après J.-C.) qui semblent tous indiquer une claire rupture avec les traditions antérieures (Bouchard 1984 ; Guinea, 1984 ; de Boer, Tolstoy, 1989).

Par contre, selon des fouilles récentes dans l'île de La Tolita, les monticules dont l'île tire son nom seraient antérieurs à tous ces autres monticules (Valdez, 1987).

\section{- De la Conquête à l'époque moderne}

Lors de l'arrivée des européens, il semble que survivaient encore certaines traditions. A La Tolita même, subsistaient des garnisons indigènes qui prétendaient ne pas être originaires de cette île mais assurer la garde de ce lieu toujours important culturellement pour eux (Cabello de Balboa, 1945). On ne peut toutefois savoir si l'ancienne métropole tenait encore un rôle actif dans les échanges commerciaux et culturels ou si on la gardait, bien qu'elle ait été désertée, en souvenir d'une époque déjà lointaine et révolue.

Au cours de la période "coloniale", la région, jugée hostile et inhospitalière pour les européens, resta longtemps marginalisée. Les quelques tentatives espagnoles militaires ou évangéliques se soldèrent par presque autant de cuisants échecs. 
Ce n'est qu'à l'époque moderne que la région pu s'ouvrir vers l'intérieur par la création de voies très précaires reliant directement la côte à la "sierra". Malgré cela et en contraste avec l'époque florissante préhispanique, aussi bien en Équateur qu'en Colombie le monde industriel demeure impuissant à mettre en valeur ces régions qui abritent surtout des populations afro-américaines comptant parmi les plus pauvres des sociétés modernes.

\section{L'ÉCONOMIE DES GROUPES PRÉHISPANIQUES}

L'absence d'évidences d'occupation antérieure au milieu du premier millénaire av. J.-C. nous permet d'envisager que cette région, jusqu'alors probablement inhabitée, fut peuplée, vers 600 av. J.-C., par des groupes culturels connaissant déjà l'agriculture et la poterie. Ces populations préhispaniques se trouvèrent alors face à un milieu naturel bien différent de celui du reste de la côte équatoriale. Pour s'implanter durablement il leur fallut s'adapter à un milieu naturel très particulier où l'eau est un élément majeur. Ainsi, outre le fait que la plupart des déplacements doivent se faire par voie aquatique, et non par voie terrestre, une grande partie de l'alimentation peut être tirée de la mer ou des fleuves.

Les premiers groupes connus dans cette région surent tirer parti du grand potentiel de pêche qu'elle offre. De nombreux poids de filets, découverts dans les anciens niveaux archéologiques (et dans les couches postérieures), sont le principal indice de cette pêche, qui fait encore vivre de nos jours la majeure partie de la population.

La pêche de poissons, en mer ou dans les estuaires, et de crevettes du genre Peneidés très abondantes dans cette région apportait l'essentiel des protéines animales. Cette pêche de poissons et de crustacés est sujette aux variations saisonnières. Pendant les périodes où cette pêche diminue, la récolte de coquillages marins comestibles compense ce manque en protéines. Parmi ceux-ci, figurent les huitres de palétuviers mais surtout des bivalves semblables aux palourdes et aux coques. Enfin, la pêche aux crabes de palétuviers ainsi que celle des poissons-chats dans les eaux douces des rivières complètent ces moyens de subsistance régionaux.

Dans les moyens de subsistance qui relèvent de la prédation, les activités de collecte de végétaux dans la forêt proche durent également jouer un rôle important, bien que la disparition des vestiges végétaux dans ce milieu limite les certitudes sur ce point. De très nombreux arbres fruitiers tropicaux poussent à l'état sauvage et donnent en abondance des fruits comestibles. Avocats, anones, goyaves, papayes sont les plus connus. On trouve aussi divers palmiers, dont les tiges ou les fruits en régimes sont souvent de haute valeur nutritive. Les pépins et graines de ces fruits, récoltés dans la forêt et consommés dans les sites, peuvent germer spontanément dans les aires d'évacuation de déchets. II est probable que ce phénomène permit une proto-arboriculture, non intentionnelle au départ.

Enfin, nous disposons de plusieurs indices qui nous permettent de supposer que, malgré les difficultés d'un milieu a priori peu favorable, cette économie était renforcée par une agriculture déjà développée. Plusieurs instruments de mouture (meules et mains de meules) sont considérés comme l'indication indirecte de la culture de céréales. La culture du maïs existe pour des époques bien antérieures dans les régions voisines. II est légitime de supposer que les populations préhispaniques du littoral nord-équatorial, issues de la même tradition formative, consommaient le maïs qu'elles cultivaient sur les terres les plus appropriées. Nous pouvons aussi supposer la culture de plantes végétatives, en particulier le manioc, au moins dans sa variété "douce", directement consommable.

Alors que, dans les régions plus sèches et aux sols plus riches, il est possible de concentrer les surfaces cultivées, les conditions spécifiques du littoral nord-équatorial ne permettent pas une économie exclusivement agraire. En effet, une forte concentration de cultures créerait un risque trop important de maladies et de parasites des plantes en raison du climat équatorial chaud et humide. La meilleure prévention naturelle reste encore de ne pas trop rapprocher les aires cultivées afin d'éviter que la contagion gagne l'ensemble des plantations. On doit donc ne cultiver qu'une petite partie du territoire habité et maintenir un écart assez vaste entre les plantations. En outre, la productivité se trouve limitée par une pauvreté générale des sols aux faibles capacités de regénération. Ces conditions rendent presque impossibles des récoltes excédentaires et la constitution de réserves alimentaires, au demeurant très difficiles à conserver dans un tel climat. II est donc nécessaire d'obtenir par prédation des aliments frais que la production agricole ne peut prétendre fournir à elle seule. Si les milieux terrestres permettent cette prédation, surtout pour obtenir des aliments végétaux, seul le milieu aquatique offre des ressources protéiniques abondantes, variées, et renouvelables presque indéfiniment, si l'on respecte le seuil de reproduction naturelle.

La limitation des activités agricoles apparaît comme un trait caractéristique de cette économie régionale car elle oblige à conserver une grande place à la prédation.

Mise au point dès la Période Formative finale, cette économie semble avoir eu une grande influence sur les modèles d'établissement régionaux. Les sites d'habitat ont été surtout découverts en bordure ou à proximité des cours d'eau inférieurs, montrant que les populations préhispaniques cherchaient à s'établir en position "de seuil", à la limite de la mangrove et de la forêt. Une telle position stratégique permet aux habitants d'exploiter les deux milieux en les plaçant au plus près possible des endroits de pêche et de culture et en instaurant donc un équilibre optimal entre les modèles d'établissement et les modes de subsistance propres à ces groupes de la région littorale.

\section{LES ÉCHANGES RÉGIONAUX : NAVIGATION ET FRÊT MARITIMES}

Pour aborder ce thème, il est indispensable de faire l'analyse des modalités pratiques des déplacements à l'intérieur de la région du littoral nord-équatorial. On ne peut exclure complètement l'hypothèse de déplacements terrestres, possibles au prix de grands efforts et très lents en raison des détours obligatoires. 
Dans le cas de courts déplacements locaux, on peut aussi se déplacer par voie aquatique en utilisant le réseau de fleuves et même passer d'un cours d'eau à l'autre par des chenaux de marée qui les interconnectent à marée haute. La navigation maritime, plus ou moins loin des côtes, peut aussi avoir joué un rôle essentiel dans les contacts entre les diverses zones d'habitat préhispaniques de la région littorale.

L'expérience actuelle démontre la grande supériorité des déplacements aquatiques sur les transports terrestres. C'est en réalité l'unique moyen efficace et rapide de déplacement et de transport de fret dans cette région où les rivières et les marécages sont autant d'obstacles. Les entraves naturelles à la facilité de communication entre le sud et le nord impliquent néanmoins le réexamen des conditions d'une telle navigation côtière, attestée dans le reste du littoral équatorial dès le Formatif ancien (Holm, 1984).

Ce sujet, d'ailleurs déjà abordé (Alcina Franch, Alonso, Bouchard, Guinea, 1987), passe d'abord par l'étude critique des embarcations utilisées et de celles des conditions pratiques d'utilisation en mer, à partir des observations sur le terrain et des quelques indications archéologiques et ethnohistoriques disponibles.

Pour l'époque préhispanique les embarcations connues sur cette côte équatoriale se résument à deux types : les radeaux (en espagnol, balsas) et les pirogues (en espagnol, canoas ). Deux sortes de documents, assez peu nombreux, les attestent : les commentaires des Espagnols au moment de la Conquête et les évidences archéologiques. Dans la région du littoral Pacifique nord-équatorial, les Espagnols ne mentionnent que l'emploi de pirogues monoxyles de tailles diverses et non de grands radeaux élaborés. Toutefois, de précaires plates-formes flottantes servaient d'embarcations temporaires en eaux calmes, montrant que le principe du radeau était connu bien qu'il ne fut pas appliqué dans la région pour de grandes embarcations navigant en mer.

Outre ces témoignages nécessairement tardifs, pour cette région nord-équatoriale les seules évidences sont quelques représentations en céramique de pirogues et de

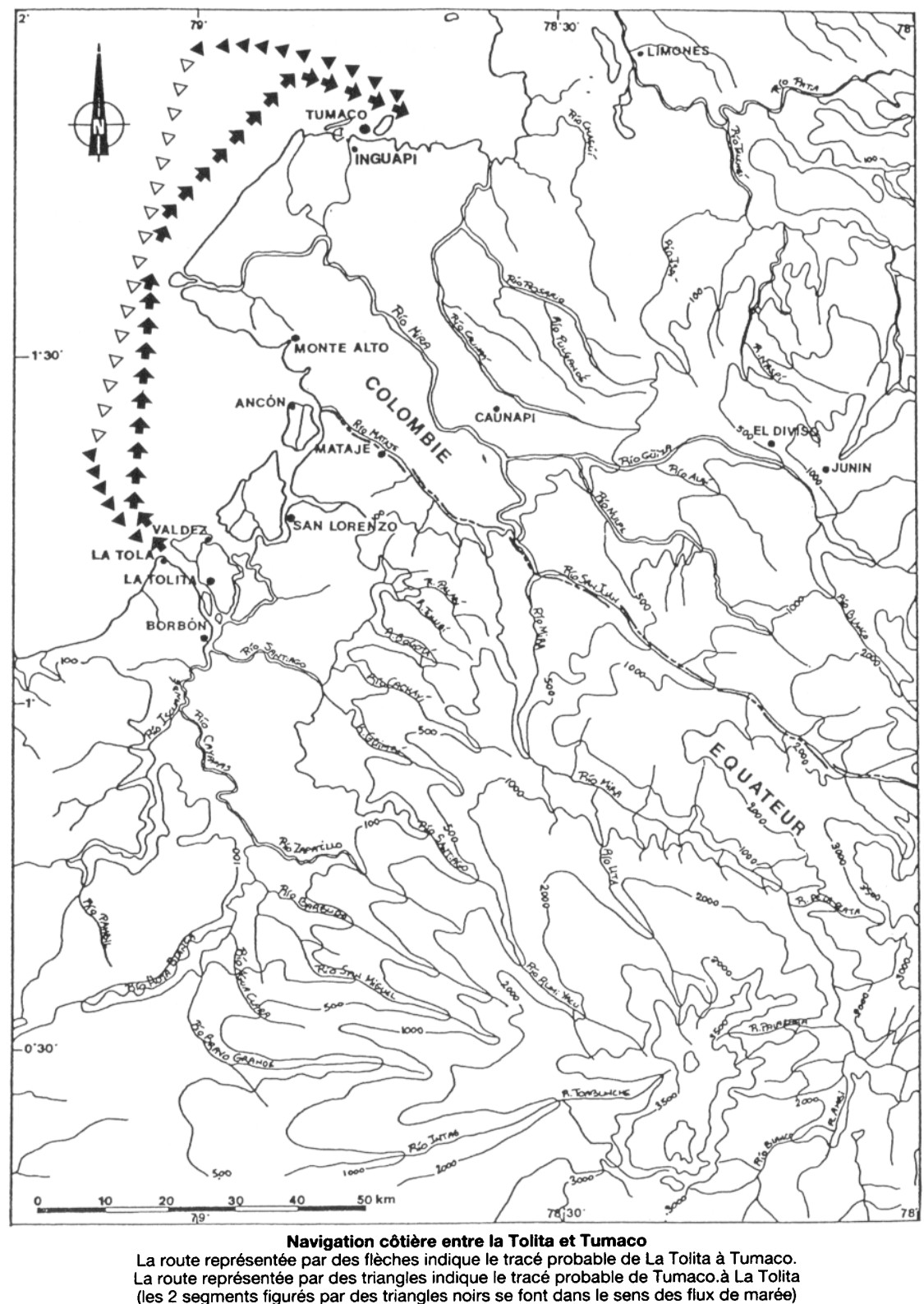

pagayeurs parmi les figurines dites "La Tolita-Tumaco", datables de la période des Développements Régionaux. La pratique de la navigation maritime ne peut être mise en doute pour ces groupes préhispaniques, mais il nous faut donc n'envisager que l'usage de pirogues et non celui de radeaux, comme c'était le cas plus au sud.

Par ailleurs, l'examen détaillé du régime des vents et des courants de surface dans cette région au moyen des documents modernes de navigation (cartes marines et les Pilot charts of the South Pacific Ocean) révèle une très forte prédominance de vents et de courants marins favorables à un voyage du sud vers le nord et, donc, défavorables de façon quasi permanente au voyage du nord vers le sud.
Si le voyage sud/nord ne posait donc aucun problème majeur, le retour semble plus problématique et implique donc l'examen détaillé de la "faisabilité" d'échanges par voie maritime entre les régions de La Tolita et de Tumaco. Celle-ci dépend des courants marins de surface et des vents dominants dont les caractéristiques sont demeurées stables jusqu'à nos jours. Leur étude en est donc possible grâce à des documents modernes de navigation, telles les "chartes de pilotes" publiées pour le Pacifique Sud par la Defense Mapping Agency (Washington). Ces chartes rendent compte de l'état moyen très précis des orientations des vents et courants, détaillés mois par mois pour des secteurs de navigation. Leur consultation permet de faire la synthèse des conditions pour le littoral Pacifique nord-équatorial. 
On doit noter que, pendant trois mois (Septembre, Octobre, Novembre), il existe un fort courant de 15 à 20 nœuds marins en sens sud/nord, qui rend très difficile une route directe à contre-courant, surtout si les vents soufflent aussi du sud vers le nord, comme c'est le plus fréquent tout au long des douze mois de l'année. Ce même courant sud/nord s'exerce encore, mais plus faiblement, pendant 6 autres mois (de Mars à Août compris). Pendant les derniers mois (Décembre, Janvier, Février) prédomine un courant allant de la terre vers le large (c'està-dire est/ouest). Les vents dominants dans cette région sont plutôt faibles mais sont pratiquement toujours plus favorables à la navigation depuis le sud vers le nord.

Le plus souvent, donc, l'ensemble de ces conditions (courants et vents portants) facilite une navigation depuis le sud vers le nord (c'est-à-dire depuis la région de La Tolita vers Tumaco) et il défavorise la navigation en sens contraire. L'orientation des flèches marines et l'érosion des cordons littoraux façonnés par la houle, traduisent d'ailleurs sur le profil de la côte actuelle ces conditions globales.

Ces conditions font que le voyage à l'aller peut s'effectuer très directement en faisant coïncider la route prise avec la direction des courants et des vents, portant tous deux vers la région nord. En revanche, un voyage par mer depuis Tumaco vers La Tolita est rendu plus lent et implique aussi un plus grand effort de propulsion, pour compenser la force adverse des courants et les vents. Cette difficulté s'accroît d'autant plus si les embarcations portent une cargaison qui les alourdit et dont le volume fait prise aux vents contraires.

Ce retour nord/sud doit donc se faire en intégrant tous ces paramètres défavorables et y répondre en évitant de naviguer contre vents et courants, au moins jusqu'au passage du cap Manglares. Un retour selon la voie "directe" en coupant au plus court, bien qu'apparemment idéal, semble exclu pour des raisons comme la difficulté de naviguer contre vents et courants ou celle de franchir les passages difficiles près des embouchures de fleuves.

De nos jours, les pirogues motorisées utilisent des moteurs de très grande puissance pour naviguer contre le courant et le vent qui rendent inefficaces les moteurs moins puissants.

Pour effectuer ce voyage dans le sens nord/sud il est donc nécessaire de suivre une route "indirecte" (qui réduit le temps de navigation contre le courant et contre le vent). Celle-ci se décompose en trois segments successifs. Le premier segment se fait en s'éloignant d'abord de la côte vers l'ouest puis un second segment se fait en mettant le cap vers le sud jusqu'à la hauteur des ports d'arrivée qui sont atteints en navigant vers l'est pendant le troisième et dernier segment.

Pour diminuer encore ces conditions défavorables, un "programme optimisé" de navigation consiste à effectuer le premier et le dernier segment avec l'aide du flux des marées descendantes et montantes. En effet, comme dans toutes les régions à fort marnage, ces courants sont toujours très puissants près des côtes et il importe de ne pas naviguer à contre-courant de flux ou de reflux. II faut pour cela quitter la rade de Tumaco au moment où la marée se retire et gagner le large avec le flux descendant, puis faire route au sud pendant l'étale de basse mer et entrer dans l'embouchure du Santiago avec le flux montant (carte 2 ).

Une telle stratégie de navigation peut s'établir sur un cycle complet de marée ou sur tout autre multiple du cycle. L'utilisation judicieuse des flux de marée permet ainsi d'accroître la vitesse dans le premier et dans le dernier segment du trajet, tout en réduisant l'effort des pagayeurs qui peuvent ainsi conserver leurs forces pour le segment intermédiaire qui doit nécessairement se faire à contre-courant et contre le vent.

II est par ailleurs important d'examiner en détail les figurines en céramique de ces pagayeurs attribuées à la culture "La Tolita-Tumaco" dans les collections de musée et dont plusieurs sont publiées (d'Harcourt, 1942 ; Sanchez Montañez, 1981). Elles présentent une particularité remarquable : le visage porte une boule en pastillage sur une des joues indiquant l'utilisation de chique par ces pagayeurs. Cette pratique peut être rapprochée de l'usage de chiques de coca très souvent utili- sées pendant l'effort par les populations traditionnelles préhispaniques ou actuelles des Andes. Dans ce cas, il est probable qu'un tel stimulant (coca ou autre) permettait aux marins de pagayer sans arrêt lors du segment de navigation à contre-courant et contre le vent, jusqu'au moment où le flux montant les propulsait vers la côte.

Ainsi résumée cette navigation de cabotage entre les deux régions peut paraître simple, mais elle implique toutefois une longue pratique de la navigation qui ne peut être le fait que de marins expérimentés, même si ces navigations se limitaient à des distances relativement courtes. Elle permet aussi de comprendre les emplacements préférentiels des établissements dans les embouchures comme à La Tolita Pampa de Oro, ou à La Tolita de Los Ruanos.

Elle explique aussi le choix très rationnel de l'établissement d'un port de transit sur la côte bien protégée de l'île El Morro, à l'entrée de la rade de Tumaco, face au continent. Ce port permettait en effet de se trouver au plus près de la mer pour partir directement vers La Tolita dès que la marée descendait et, ensuite, prendre la route du sud. Avant sa destruction, lors de l'aménagement du port moderne, ce site aurait atteint $1,5 \mathrm{~km}$ de long, ce qui témoigne de son importance à l'époque (Cubillos, 1955). Si on prend en considération le temps nécessaire pour traverser la baie depuis les fleuves qui y débouchent, le gain de temps ainsi réalisé paraît considérable et permettait ainsi de faire en temps opportun le premier segment du voyage nord/sud. En outre, il semble probable qu'il servit de relais pour atteindre la région de Guapi, au nord, en supposant une navigation reproduisant celle du trajet Tumaco-La Tolita.

Les difficultés représentées par les conditions de navigation nord/sud nous font supposer qu'il fallait à cette époque des impératifs très forts pour établir cette relation micro-régionale. II nous semble clair que cette navigation de cabotage interdisait pratiquement un transport de charges lourdes, volumineuses ou périssables. L'étude globale de la région proche de La Tolita (en y incluant tout le cours moyen du rio Cayapas-Santiago) a montré que les groupes préhispaniques devaient disposer sur place de ressources ali- 
mentaires en quantités suffisantes pour leur permettre une auto-suffisance. Aux alentours de La Tolita, des systèmes de cultures sur billons, ou "camellones", ont été retrouvés en grand nombre. Cela nous permet d'exclure l'hypothèse d'un trafic de produits alimentaires, peu compatible avec un transport maritime par pirogue.

Les raisons de ces échanges nord/sud doivent donc être trouvées ailleurs et tout laisse supposer qu'ils portaient sur des biens à la fois très valorisés et peu encombrants, comme l'or et, peut-être, d'autres matières premières précieuses. Celles-ci auraient été ramenées à la Tolita et sa région pour y être transformées et éventuellement réexportées dans les territoires où s'exerçait I'hégémonie de La Tolita. Les études de composition métallique de l'orfévrerie ont montré que l'or vient bien des alluvions aurifères du littoral nord-équatorial, aussi bien dans l'extrême nord de l'Équateur que dans le sud de la Colombie.

Comme le prouve leur découverte dans des sépultures, la plupart des objets en or étaient déposés avec les corps des défunts les plus importants, soit comme offrandes, soit en tant qu'objets personnels qu'ils portaient de leur vivant.

Cet usage implique qu'il fallait sans cesse obtenir plus de matière première pour alimenter les artisans orfèvres. II est vraisemblable qu'un des principaux motifs de l'expansion vers le nord, à partir de La Tolita, fut la recherche d'alluvions aurifères pour répondre à la demande incessante de matière première pour produire ces objets disparaissant dans les sépultures.

Une autre hypothèse expliquerait aussi la pauvreté en orfèvrerie et l'absence de sépultures avec des vestiges précieux pour toute la région la plus au nord, comme l'a d'ailleurs confirmé notre propre expérience dans la région de Tumaco.

Alors qu'à La Tolita de nombreuses sépultures ont livré une somme incalculable d'objets précieux, l'absence de sépultures contenant d'importantes offrandes laisse supposer qu'il n'y avait pas d'autres lieux funéraires préhispaniques dans la région nord. Comme l'a suggéré Valdez, une partie des activités de La Tolita devait être cen- trée sur les rites funéraires et sur la fabrication d'objets liés à ces rites (Valdez, 1986). II nous semble qu'on peut même suggérer que cette île constituait en fait l'unique nécropole de cette civilisation, réservée aux plus importants défunts. Ce grand centre funéraire structurait, sans doute, toute une vaste région côtière allant de La Tolita à Tumaco, et les territoires de l'intérieur accessibles par les fleuves, mais qui put s'étendre depuis Esmeraldas (au sud) jusqu'à Guapi (au nord) où furent aussi découverts des vestiges de cette "culture Tumaco-La Tolita" (Patiño Castaño, 1987).

Cette fonction funéraire permet ainsi de présumer qu'une puissante tradition exigeait de rassembler ces défunts dans un endroit unique, constituant leur terre d'origine culturelle, ce qui impliquait donc de les rapatrier après leur mort à La Tolita.

Il faut pour cela porter attention aux représentations anthropomorphes en céramique de La Tolita. Des "plaques" moulées en céramique, représentant un homme ou une femme allongés sur le dos et immobilisés par des bandelettes, ont fait l'objet d'interprétations diverses (Lehmann, 1951; d'Harcourt, 1942 ; Sanchez Montañez, 1979). Selon celles-ci, elles représenteraient des individus pendant des rites d'initiation ou encore des patients préparés pour subir une opération chirurgicale. Toutefois ces interprétations négligent une hypothèse selon laquelle ces plaques correspondraient plutôt à des défunts. Leur position étendue sur une civière évoque fortement des personnages sans vie, qui semblent ainsi préparés pour être transportés à La Tolita et inhumés selon la coutume du retour à la terre d'origine culturelle.

On comprend alors d'autant mieux l'usage de substances stimulantes et anxyolitiques par les marins chargés d'un tel transport : aux dangers propres à la navigation en mer s'ajoutait celui d'être accompagné par un défunt, autrefois puissant et peut-être encore considéré comme doté de forces supérieures. Les marins spécialisés dans cette double tâche se voyaient ainsi confier la charge de deux biens figurant parmi les plus valorisés : l'or pour fabriquer les insignes de pouvoir et les offrandes funéraires et les défunts puissants qu'il fallait ramener à la né- cropole pour y accomplir les rites funéraires.

De telles hypothèses, qui demeurent certes difficiles à démontrer, permettent cependant de supposer une civilisation particulièrement soucieuse d'honorer dans la vie comme dans la mort ses chefs politiques ou religieux les plus importants. L'objet du trafic maritime ne se limitait peutêtre pas seulement à la quête d'or et à l'impératif rituel d'ensevelir les puissants dans la nécropole de La Tolita, mais les évidences archéologiques incitent à penser que ces deux activités sous-tendaient fortement la société de l'époque. II est probable que tant que dura l'hégémonie de La Tolita, la fonction de nécropole contribua à unir la sphère d'influence culturelle autour de tels rituels funéraires.

Selon le témoignage de Cabello de Balboa qui rapporte qu'une garnison d'une centaine d'indiens occupait l'île où on venait encore apporter des offrandes de poudre d'or et effectuer des prières (Cabello de Balboa, 1945) le souvenir de la vieille nécropole survécu bien au-delà du déclin de l'hégémonie de La Tolita. Malgré les nombreux siècles écoulés depuis l'époque de son apogée culturelle, l'île restait un important site funéraire sacré, toujours respecté et entretenu par les groupes indigènes contemporains de la conquête qui conservaient ainsi un lien étroit avec leur lointaine origine.

Cette vénération ne tarda pas toutefois à prendre fin : dès le début du XVIII', fray Juan de Santa Gertrudis faisait état de destructions des sépultures de l'île pour y récupérer les précieuses offrandes funéraires (fray Juan de Santa Gertrudis, 1970).

\section{CONCLUSION}

A partir d'un territoire potentiel allant de l'océan aux cordillères et dont les extrêmes limites (haute mer, piémont et bas versants) ont pu être exploitées par incursions pour se procurer soit des produits de pêche soit des matières premières (or et obsidienne, entre autres) nous tendons à considérer un territoire plus réduit, d'incursions régulières pour des activités de pêche (mer proche des côtes, estuaires et cours d'eau) et de prédation terrestre (mangrove et forêt de la plaine alluviale inté- 
rieure) ainsi qu'un territoire proprement de résidence, relativement restreint, correspondant à d'éventuels sites d'habitat sur les cordons littoraux aujourd'hui disparus et dans une étroite frange localisée au contact de la mangrove et de la forêt, à l'extrémité de la plaine alluviale, où furent découverts la plupart des sites. II faut aussi sans doute inclure comme territoire de résidence les berges de certains cours d'eau colonisées par des groupes paraissant venus du littoral.

Ce n'est que par un tel cumul d'exploitation de secteurs si différents que ces groupes purent atteindre le niveau de développement dont les vestiges archéologiques sont le reflet. Cette "extension à l'horizontale", qui cherche à multiplier les milieux exploités à partir d'un foyer d'habitat préférentiel, se rapproche ainsi de celle qui est définie pour les hautes cultures andines sous le nom "d'économie verticale".

Bien qu'ils se soient développés dans un environnement de forêt tropicale, ces groupes du littoral Pacifique nord-équatorial se différencient ainsi des groupes selvatiques américains dont l'économie de "forêt tropicale humide" repose sur la prédation (chasse, pêche, cueillette) et/ou l'agriculture de plantes végétatives (surtout le manioc) mais sans culture de céréales.

Un tel développement culturel et économique pendant de longs siècles contraste avec les tentatives modernes de développer la plupart des régions boisées tropicales Malgré une technologie hautement développée, elles n'aboutissent le plus souvent qu'à la destruction d'un milieu naturel trop fragile pour résister aux agressions anthropiques.

L'expérience préhispanique montre un exemple de conservation de l'environnement tout en donnant aux groupes humains qui l'habitaient des ressources bien supérieures à celles des populations actuelles qui comptent parmi les plus déshéritées du litttoral Pacifique équatorial.

\section{Bibliographie}

Alcina-Franch J., Alonso A., Bouchard J.F., GUINEA M. (1987) - Navegación precolombina : el caso del litoral pacífico ecuatorial : evidencias e hipótesis.
Revista Española de Antropología Americana. $\mathrm{N}^{\circ} \mathrm{XVII}$, p. 35-73.

BouchaRd J.-F. (1979) - Hilos de Oro Martillado en la Costa Pacífica del Sur de Colombia. Boletín del Museo del Oro, Año 2, mayo-agosto, p. 21-24.

BOUCHARD J.-F. (1984) - Recherches Archéologiques dans la Région de Tumaco (Colombie). Mémoire $n^{\circ} 34$; Institut Français d'Études Andines. Éditions Recherche sur les Civilisations. A.D.P.F. Paris.

BoUCHARD J.-F. (1986) - Las más antiguas culturas precolombinas del Pacífico ecuatorial septentrional. Simposio del $45^{\circ}$ Congreso Internacional de Americanistas : Arqueología y etnohistoria del sur de Colombia y norte del Ecuador. Miscelanea Antropológica Ecuatoriana. $N^{\circ} 6$, p. 109-129.

BOUCHARD J.-F. (1989) - Evidencias de relaciones interculturales en la región norte (sur de Colombia y norte del Ecuador). Relaciones interculturales en el área ecuatorial del Pacifico durante la época precolombina. Proceedings 46 Congreso Internacional de Americanistas (Amsterdam, 1988). B.A.R. International Series $\mathrm{N}^{\circ} 503$, p. 29-44. Oxford.

Cabello Balboa M. (1945) - Verdadera descripción y relación de la provincia y tierra de las esmeraldas. In Obras de Miguel Cabello Balboa Vol. 1, p. 1-76. Quito (1583).

Cubillos J.C. (1955) Tumaco - notas arqueológicas. Ministerio de Educación. Bogotá.

DeBoer W. Tolstoy P. (1989) - An archaeological sequence for the Santiago-Cayapas River basin, Esmeraldas, Ecuador. Journal of field archaeology. Vol. 16, $\mathrm{N}^{\circ} 3$, fall 1989, p. 295-308.

GUINEA M. (1984) - Patrones de asentamiento en la arqueología de Esmeraldas (Ecuador). Memorias de la Misión Arqueológica Española en Esmeraldas. Vol. 8. Ministerio de Asutos Exteriores. Madrid.

GUINEA M. (1986) - El Formativo de la región sur de Esmeraldas visto desde el yacimiento de Chévele. Simposio del $45^{\circ}$ Congreso Internacional de Americanistas : Arqueologia y etnohistoria del sur de Colombia y norte del Ecuador. Miscelanea Antropológica Ecuatoriana. $\mathrm{N}^{\circ} 6$, p. 19-46.

Harcourt (D') R. (1942) - Archéologie de la province d'Esmeraldas, Équateur. Journal de la Société des Américanistes. $\mathrm{N}^{\circ} 34$, p. 61-200.

LEHMANN H. (1951) Le personnage couché sur le dos : sujet commun dans l'archéologie du Mexique et de l'Équateur. Proceedings of the 29th international Congress of Americanists ; vol. 1, p. 291-298. Chicago.

MARCOS J. (1992) The neolithic revolution in north western south America: emerging patterns of specialization at
Real Alto. Symposium in memory of Donald Lathrap. American Anthropological Association. San Francisco. (sp)

MONTAÑo M.C. (1991) - La subsistencia en La Tolita : un enfoque etnoarqueológico. Ms. in Congreso "10 años de arqueologia ecuatoriana”.

Montaño M.C. (1991) - El manejo de los recursos naturales en La Tolita en su etapa clásica. Ms. in Encuentro "Investigaciones arqueológicas de culturas comunes de la costa Pacifica". Esmeraldas.

Patiño Castaño D. (1987) - Arqueología de la costa pacífica caucana. Boletín de arqueología. Año 2, $\mathrm{N}^{\circ} 1$, p. 65-81. Fundación de Investigaciones arqueológicas nacionales.

Patiño Castaño D. (1988) - Asentamientos prehipanicos en la costa Pacifica caucana. Fundación de Investigaciones arqueológicas Nacionales. Banco de la República. Bogotá.

REICHEL-DOLMATOFF G. (1987) - Arqueología de Colombia : un texto introductorio. Fundación Segunda Expedición Botánica. Bogotá.

Sanchez Montañes E. (1979) - Un tipo de placa ritual de la cultura Tumaco La Tolita. Actes du 42 Congrès International des Américanistes. Vol. 9A, p. 273281. Paris.

SANTA GertRUdis fray J. (1970) - Maravillas de la naturaleza. T. III. Biblioteca popular. Bogotá.

ScotT D. A. (1986) - Gold and silver alloy coatings over copper; an examination of some artefacts from Ecuador and Colombia. Archaeometry. Vol. 28, p. 33-50.

UHLE M. (1927) - Las antiguas civilisaciones esmeraldeñas. Anales de la Universidad Central. (Quito). Vol. 38, $\mathrm{N}^{\circ} 259$.

UHLE M. (1927) - Estudios esmeraldeños. Anales de la Universidad Central. (Quito). Vol. 39, N²62, p. 1-61.

VALDEZ F. (1986) - Investigaciones arqueológicas en La Tolita (Esmeraldas). Simposio del $45^{\circ}$ Congreso Internacional de Americanistas : Arqueología y etnohistoria del sur de Colombia y norte del Ecuador. Miscelanea Antropológica Ecuatoriana. $N^{\circ} 6$, p. 81-107.

VALDEZ F. (1987) - Proyecto arqueológico La Tolita. Banco Central del Ecuador. Quito.

VALDEZ F. (1989) - L'évolution démographique dans les mangroves de la côte nord-ouest de l'Équateur, aux périodes formative et de développement régional. In Equateur 1986. Vol. 1, p. 291-308. ORSTOM. Paris.

Valdez F., Montaño M.C., Adoum R., Leiva S., Yepes A. et RodRiguez E. (1989) Nuestro pasado : La Tolita. Museo del Banco Central del Ecuador. Quito.

Jean-François BOUCHARD C.N.R.S.-UPR 312 , Archéologie de l'Amérique Préhispanique, Musée de l'Homme, 75116 Paris 